Bio - grafía. Escritos sobre la Biología y su Enseñanza. ISSN 2027-1034

Edición Extraordinaria. p.p. 857 - 864

Memorias del IX Encuentro Nacional de Experiencias en Enseñanza de la Biología y la

Educación Ambiental. IV Congreso Nacional de Investigación en Enseñanza de la Biología.

\title{
ENSEÑAR Y APRENDER SOBRE LAS FUNCIONES VITALES DESDE EL ENFOQUE DE LA MODELIZACIÓN. DOS ESTUDIOS DE CASO CON ESTUDIANTES DE QUINTO GRADO DE PRIMARIA
}

TEACH AND LEARN ABOUT VITAL FUNCTIONS FROM THE MODELING APPROACH. TWO CASE STUDIES WITH FIFTH GRADE STUDENTS

\author{
Liceth Natalia Alzate Martínez \\ Silvia Farley Jaramillo Carrillo \\ Dra. Fanny Angulo Delgado ${ }^{2}$
}

\section{RESUMEN:}

Este artículo tiene la finalidad de mostrar los fundamentos del proceso de modelización implementados en el aula de clase, mediante la aplicación de una secuencia de enseñanza y aprendizaje que sirvió como hipótesis al interrogante: ¿Cómo evoluciona el modelo estudiantil inicial hacia el modelo científico escolar de arribo, en torno de la enseñanza de las funciones vitales, con estudiantes de quinto grado de primaria? Los análisis de los resultados se realizaron con base en el modelo científico ONEPSI propuesto por Rufina Gutiérrez (2014), donde se observa la transformación paulatina del concepto de ser vivo de dos instituciones educativas durante las cuatro fases de la secuencia, observando el cambio entre el modelo estudiantil inicial (MEi) y el modelo científico escolar logrado (MCEL).

PALABRAS CLAVES: Modelización, modelo ONEPSI, secuencia de enseñanza y aprendizaje, modelo estudiantil inicial, modelo científico escolar de arribo, modelo científico escolar logrado.

\section{ABSTRACT:}

This article aims to show the fundamentals of the modeling process implemented in the classroom, by applying a sequence of teaching and learning that served as a hypothesis to the question: How does the initial student model evolve towards the scientific model? Around the teaching of the vital functions, with students of fifth grade of primary? The analysis of the results was based on the scientific model ONEPSI proposed by Rufina Gutiérrez (2014), where we observe the gradual transformation of the concept of living being of two educational institutions during the four phases of the sequence, observing the

\footnotetext{
${ }^{1}$ Estudiantes programa maestría en educación, Universidad de Antioquia. Correo electrónico: pkeniko@hotmail.com y sifajacar@gmail.com

${ }^{2}$ Doctora en Didáctica de las Ciencias. Profesora, Universidad de Antioquia. Medellín - Colombia. Correo electrónico: fanny.angulo@udea.edu.co
} 
Bio - grafía. Escritos sobre la Biología y su Enseñanza. ISSN 2027-1034

Edición Extraordinaria. p.p. 857 - 864

Memorias del IX Encuentro Nacional de Experiencias en Enseñanza de la Biología y la

Educación Ambiental. IV Congreso Nacional de Investigación en Enseñanza de la Biología.

change between the Initial student model (MEI) and the academic school model achieved (MCEL).

KEYWORDS: Modeling, model ONEPSI, sequence of teaching and learning, teaching, initial student model, scientific school arrival model, school scientific model achieved.

\section{INTRODUCCIÓN}

En la etapa de primaria la educación científica es débil e incluso, puede estar ausente en la formación básica de los estudiantes (Pujol, 2003). No obstante, abordar un problema de tal magnitud solo es posible con el concurso de diferentes entidades y protagonistas, uno de los cuales es el maestro que enseña ciencias naturales. La reflexión sobre la práctica pedagógica implica incorporar elementos de orden teórico que además de dar sentido a la cotidianidad de la enseñanza y el aprendizaje de las ciencias naturales, muestra enfoques distintos que pueden ser tomados como fundamento para replantear la enseñanza en búsqueda de posibilidades de aprendizaje según las cuales, los estudiantes puedan entender el mundo que los rodea usando el conocimiento científico para interpretarlo.

Es así como en esta investigación la «Modelización en la enseñanza de las ciencias» se instala como el enfoque epistemológico desde el cual la construcción de la ciencia en un entorno de aula de primaria es una oportunidad real para los alumnos. En los casos objeto de este estudio, los niños y las niñas construyeron explicaciones acerca de la lombriz de tierra como fenómeno educativo cuyo valor radica en que les permite comprender su comportamiento como ser vivo.

Al finalizar la educación primaria (quinto grado en el sistema educativo colombiano), el currículo de ciencias naturales aspira a que los estudiantes entiendan las funciones vitales en tanto son distintivas de los seres vivos. Sin embargo, la experiencia docente insiste en señalar que pocas veces se alcanza esta meta, porque ante situaciones cotidianas en las que serían deseables explicaciones cercanas a la Biología la tendencia de respuesta de los alumnos se inclina hacia frases bien conocidas como 'los seres vivos nacen, crecen, se reproducen y mueren' y para muchos de ellos el movimiento entendido como desplazamiento es vital. Además, el comportamiento y apariencia de su propio cuerpo (humano), se configura en el referente por excelencia de 'ser vivo'. A esta descripción hay que sumarle las características que se derivan de condiciones sociológicas, antropológicas y de cultura escolar que inciden en la carencia de una motivación y compromiso personal del estudiante hacia su propia formación.

Es por ello que en esta investigación se desarrolló una propuesta didáctica donde se puso a prueba como hipótesis para dar cuenta de las posibilidades reales de construcción de una ciencia escolar con alumnos de entornos rurales o desfavorecidos socioeconómicamente, a fin de observar si los estudiantes logran evolucionar sus modelos, haciéndolos más complejos y abstractos, en cuanto incorporaron en sus 
Bio - grafía. Escritos sobre la Biología y su Enseñanza. ISSN 2027-1034

\title{
Edición Extraordinaria. p.p. 857 - 864
}

\author{
Memorias del IX Encuentro Nacional de Experiencias en Enseñanza de la Biología y la \\ Educación Ambiental. IV Congreso Nacional de Investigación en Enseñanza de la \\ Biología.
}

representaciones sobre los seres vivos entidades, propiedades y enunciados legales que explicaron el comportamiento de la vida en términos de funciones vitales.

El concepto de 'modelo científico' desarrollado por la Dra. Rufina Gutiérrez (2014, al que en adelante se mencionará como ONEPSI por la identificación de los constituyentes ontológicos, epistemológicos y psicológicos en la configuración de las representaciones tanto de los estudiantes, como de la ciencia y del currículo), se presenta en esta investigación como concepto fundamental para, por una parte, configurar los modelos estudiantil inicial, disciplinar y curricular, de cuya tensión surge el Modelo Científico Escolar de Arribo (López \& Moreno, 2014) que junto con la identificación de las demandas de aprendizaje (Leach \& Scott, 2002), se constituyen en una herramienta metodológica fundamental para el diseño de la secuencia de enseñanza y aprendizaje. Y por otra parte, el ONEPSI es el concepto básico para interpretar la evolución de los modelos estudiantiles a lo largo de la secuencia.

Se planteó responder a la siguiente pregunta, ¿Cómo evoluciona el modelo estudiantil inicial hacia el modelo científico escolar de arribo, en torno a la enseñanza de las funciones vitales, con estudiantes de quinto grado de primaria? Y para ello, se analizó la evolución del modelo estudiantil en alumnos de quinto grado de primaria, con una metodología enmarcada bajo el paradigma cualitativo, con el tipo de investigación de estudio de caso múltiple, mediante la aplicación de una secuencia de enseñanza y aprendizaje sobre las funciones vitales, en la cual, el comportamiento de la lombriz de tierra aparece como el fenómeno cuyo valor educativo radica en la posibilidad de ser modelizado por los alumnos.

La construcción de la secuencia de enseñanza y aprendizaje parte desde sus modelos iniciales, con los cuales se construyó el modelo estudiantil inicial -MEi (representaciones iniciales de los alumnos), luego se indagó el modelo curricular - $\mathrm{MCu}$ (planes de estudio y currículo), se continuó con la construcción del modelo disciplinar o científico -MD (analizando apartados del libro de Biología 5.ed, cuyos autores son Curtis y Barnes, 1993). Seguidamente, se construyó el modelo científico escolar de arribo -MCEA (como referente metodológico pero también como la meta a la que se quería llegar con los estudiantes). Luego se contrastaron estos modelos para reconocer cuales son las demandas de aprendizaje presentes en los estudiantes y así diseñar la secuencia de enseñanza y aprendizaje, la cual se desarrolló en cuatro fases. Al finalizar cada una se observó qué tanto se transformó ese modelo estudiantil hacia el MCEA (López y Moreno, 2014), con lo cual se obtuvo el modelo científico escolar logrado -MCEL (hasta dónde llegaron los estudiantes).

\section{METODOLOGÍA}

La metodología en esta investigación está enmarcada bajo el paradigma cualitativo y el tipo de investigación utilizada es el estudio de caso múltiple. El proyecto se desarrolló en 
Bio - grafía. Escritos sobre la Biología y su Enseñanza. ISSN 2027-1034

\section{Edición Extraordinaria. p.p. 857 - 864}

Memorias del IX Encuentro Nacional de Experiencias en Enseñanza de la Biología y la

Educación Ambiental. IV Congreso Nacional de Investigación en Enseñanza de la Biología.

dos instituciones educativas (I.E) oficiales ubicadas en municipios diferentes de Antioquia, estas son: I.E Rafael Núñez del municipio de Tarazá y la I.E Santa Bárbara, sede Río Abajo del municipio de Rionegro. El contexto de estas dos I.E presentan características particulares en el aspecto social, económico y cultural. Los instrumentos que se utilizaron en la recolección de la información fueron fotografías, producciones de los estudiantes y la grabación de video.

En concordancia con lo anterior se tomó el diseño de la secuencia de enseñanza y aprendizaje como una hipótesis. De acuerdo con Pujol (2003), se desarrolló en cuatro fases: 1. Verbalización de los modelos iniciales. 2. Introducción de nuevos elementos, relaciones y variables. 3. Estructuración de los modelos en construcción y 4 . Aplicación y regulación final del modelo elaborado. La figura 1 muestra el diseño de la secuencia, las actividades para cada una de las fases y las demandas de aprendizaje para cada entidad.

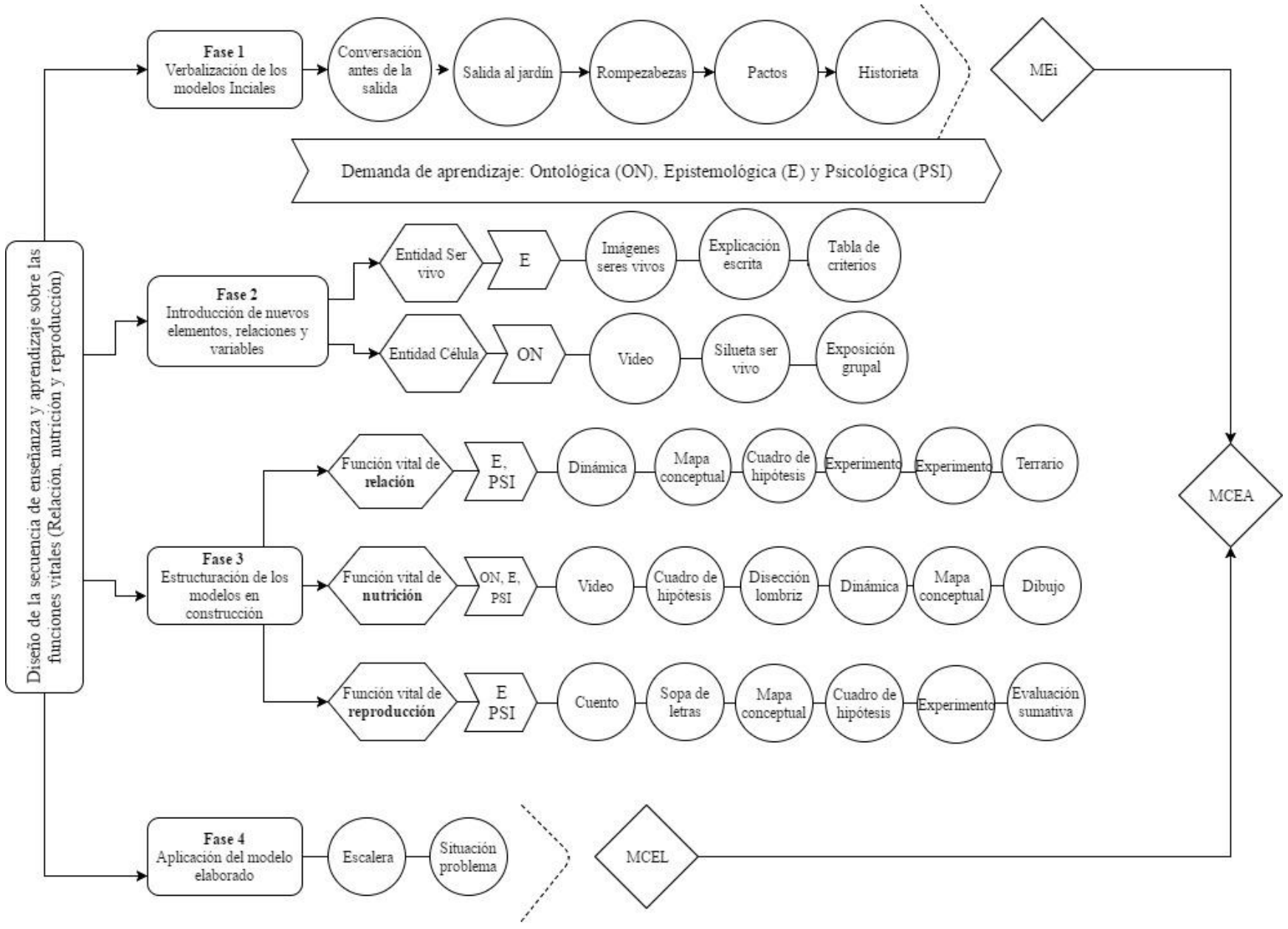


Bio - grafía. Escritos sobre la Biología y su Enseñanza. ISSN 2027-1034

Edición Extraordinaria. p.p. 857 - 864

Memorias del IX Encuentro Nacional de Experiencias en Enseñanza de la Biología y la

Educación Ambiental. IV Congreso Nacional de Investigación en Enseñanza de la Biología.

Figura 1: Diseño de la secuencia de enseñanza y aprendizaje sobre las funciones vitales. Elaboración propia a partir de Pujol (2003).

Para la realización del diseño de la secuencia de enseñanza y aprendizaje para el grado quinto sobre las funciones vitales, se hizo necesario construir diferentes modelos, desde los constituyentes ontológicos, epistemológicos y psicológicos, los cuales forman el modelo ONEPSI, ellos son: el MEi, el MCu, el Md, el MCEA y rastrear las demandas de aprendizaje de los estudiantes, para así, poder diseñar las diferentes actividades que permitieran observar la evolución de los modelos estudiantiles.

\section{RESULTADOS Y DISCUSIÓN}

Los resultados se describen de manera general para las dos instituciones educativas y según las cuatro fases de la secuencia de enseñanza y aprendizaje.

- Verbalización de los modelos iniciales

En esta primera fase de la secuencia didáctica, los estudiantes no identificaron las características propias de los seres vivos. Esto pudo deberse a que usaban su cuerpo como referente para dar explicaciones. Adjudicaron el movimiento (desplazamiento) como una cualidad que acoge a todos los seres vivos. En cuanto a la función de nutrición, argumentaron que la lombriz de tierra carecía de órganos para su digestión, asumían que tenía respiración pulmonar, que le fluía sangre, desconociendo los órganos y función del sistema circulatorio; que su piel estaba cubierta de una baba indispensable para vivir, que comía y ensuciaba tierra con la cual ayudan a las plantas. Con respecto a la función vital de reproducción, reconocían que son ovíparas, aunque desconocían que eran hermafroditas, al igual que la forma de producir sus huevos. Para la función de relación, sabían que necesitaban de un hábitat húmedo, desconociendo otras características; que el sol las dañaba, que se protegían de los depredadores y que para desplazarse se arrastraban. Con la información recolectada en esta fase se construyó el MEi, donde se evidenció que era necesario atender a nuevas demandas de tipo: ontológicas, epistemológicas y psicológicas, con el fin de lograr que los estudiantes se apropiaran de las funciones vitales como procesos que realizan todos los seres vivos.

- Introducción de nuevos elementos, relaciones y variables.

Al contrastar el MEi con el MCEA sobre el modelo de ser vivo en los estudiantes, este ha evolucionado, ya que la gran mayoría incorporaron en sus definiciones las funciones vitales, como características indispensables de los seres vivos y reconocieron que este, está formado por células, las cuales a su vez también las cumplen. Se observó que aún presentan dificultades para verbalizar en qué consistía cada una de las funciones vitales y cómo intervenían en el desarrollo y supervivencia del ser vivo. Para continuar con una 
Bio - grafía. Escritos sobre la Biología y su Enseñanza. ISSN 2027-1034

\section{Edición Extraordinaria. p.p. 857 - 864}

Memorias del IX Encuentro Nacional de Experiencias en Enseñanza de la Biología y la

Educación Ambiental. IV Congreso Nacional de Investigación en Enseñanza de la Biología.

complejización progresiva de la modelización (Gómez, 2014), es necesario atender a una nueva demanda epistemológica y psicológica que surge aquí, según la cual, los alumnos deben trascender este referente a fin de que puedan ofrecer explicaciones con un grado de complejidad y generalidad más alto con respecto a la funciones vitales.

- Estructuración de los modelos en construcción

Al contrastar el MEi con el MCEA, se pudo reconocer que el MCEL, obtenido en el desarrollo de las diferentes actividades, con los estudiantes fue: la lombriz de tierra es un ser vivo porque presenta las funciones vitales (relación, nutrición y reproducción) y está formada por células. Necesita relacionarse con el ambiente, del cual toma oxígeno del aire por la piel y una baba que la hidrata y le permite arrastrarse (repta), necesita vivir en tierra húmeda, fértil y evitar los rayos del sol, para conservar la baba. También debe protegerse de los depredadores. Para alimentarse, la boca captura los alimentos del medio y lo pasa a la faringe, luego al buche donde se acumula, para pasar hacia la molleja, donde es triturado, de allí pasa al intestino en forma de nutrientes y materia fecal [asumen que la materia fecal se forma en la molleja y no en el intestino. Es posible que esta confusión se haya mantenido porque no se aclaró oportunamente]. El intestino entrega los nutrientes al sistema circulatorio que se encarga de llevarlos hasta las células de todo el cuerpo y de recoger los desechos para eliminarlos en el ambiente, y la materia fecal al ano, donde se elimina en forma de tierra. La lombriz de tierra se reproduce cuando pone sus huevos (autoperpetuación) o recupera una parte de su cuerpo que haya perdido (autorregeneración), y es hermafrodita porque tiene los dos sexos (femenino y masculino).

- Regulación final del modelo elaborado.

Se observó al finalizar la cuarta fase de la secuencia didáctica, la transformación del MEi, ya que incorporaron en sus explicaciones las funciones vitales, pero aún sigue apareciendo el movimiento como característica de los seres vivos. Dan prioridad a sus vivencias con respecto al propio cuerpo y no a las experiencias con la lombriz de tierra. Con respecto a la función de relación, la explican como la captación de estímulos del medio para defenderse. Relacionan el concepto de nutrición, como el intercambio de materiales y energía entre el ser vivo y el medio ambiente, reconociendo los procesos necesarios para que esta función se cumpla, no obstante se requiere avanzar en el significado de la función, particularmente en lo relativo a las modificaciones del medio y al intercambio de energía (luz, calor, energía química). Y la función de reproducción es comprendida como el proceso de perduración de la especie y el de reparación de tejidos, aunque admiten que los seres unicelulares como las bacterias, también la cumplen, lo cual indica una nueva demanda de aprendizaje relacionada con la comprensión del concepto de 'tejido' y, no aparece en ningún momento la reproducción como transmisión de características genéticas a sus descendientes, es por ello que la demanda epistemológica no queda atendida.

\section{Modelo Científico Escolar Logrado - MCEL para Ambos Casos}


Bio - grafía. Escritos sobre la Biología y su Enseñanza. ISSN 2027-1034

Edición Extraordinaria. p.p. 857 - 864

Memorias del IX Encuentro Nacional de Experiencias en Enseñanza de la Biología y la

Educación Ambiental. IV Congreso Nacional de Investigación en Enseñanza de la Biología.

Para los estudiantes del grado quinto primaria de las dos instituciones educativas involucradas en el desarrollo de la presente experiencia, al finalizar la aplicación de la secuencia de enseñanza y aprendizaje de las funciones vitales, su proceso de modelización obtenido con respecto al ser vivo es: Un ser vivo se caracteriza porque está formado por células, se mueve y cumple con las funciones vitales: nutrición, relación y reproducción. Necesita alimentarse para tener energía, expulsa sus desechos como agua, baba, dióxido de carbono y recibe el aire con oxígeno. También se relaciona con el ambiente y con otros seres, necesita de un hábitat adecuado para vivir. Se reproduce cuando tiene hijos (autoperpetuación) y cuando repara alguna parte de su cuerpo dañada (autorregeneración).

En la figura 2 se hace una comparación entre el MEi y el MCEL de las dos instituciones educativas. Cabe aclarar que el MCEL se construyó contrastando el MEi con el MCEA.

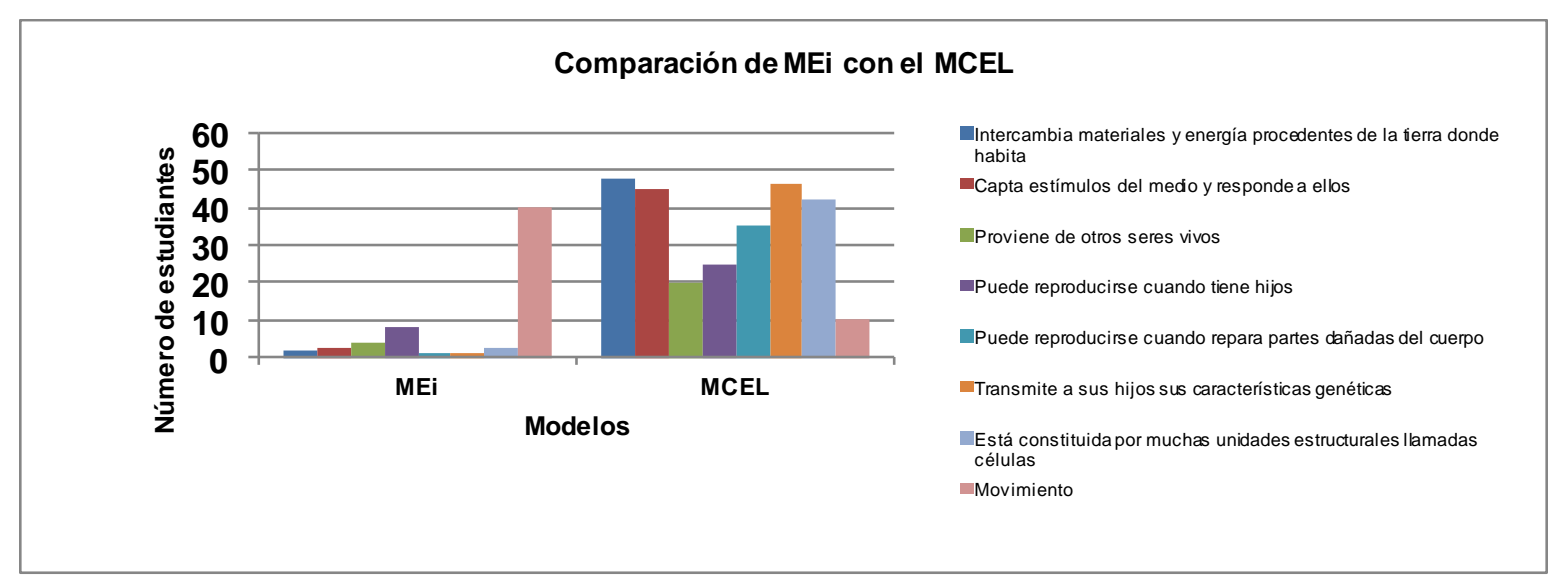

Figura 2: Comparación del modelo estudiantil inicial (MEi) con el Modelo Científico Escolar Logrado (MCEL) de dos Instituciones Educativas del departamento de Antioquia.

\section{CONCLUSIÓN}

Con respecto al análisis de la evolución de los modelos estudiantiles se deriva que el enfoque de la modelización, y en particular el concepto del modelo científico ONEPSI, son útiles para hacer las interpretaciones de dicha evolución. Enlazado con las demandas de aprendizaje, permitieron identificar los tipos de demanda de aprendizaje presentes en los estudiantes (ontológicos, epistemológicos y/o psicológicos), además se establecieron diferencias puntuales en cada etapa de la construcción del modelo, lo que permitió validar el diseño de la secuencia de enseñanza y aprendizaje sobre las funciones vitales con estudiantes del grado quinto, se implementaron simultáneamente las mismas actividades en las dos instituciones educativas, comparándose los resultados, los cuales fueron muy similares, ya que los estudiantes incorporaron en sus explicaciones las entidades: ser vivo, célula, materiales, energía, medio, estímulo, respuesta, tejido e hijo. Al explicar cómo 
Bio - grafía. Escritos sobre la Biología y su Enseñanza. ISSN 2027-1034

Edición Extraordinaria. p.p. 857 - 864

Memorias del IX Encuentro Nacional de Experiencias en Enseñanza de la Biología y la Educación Ambiental. IV Congreso Nacional de Investigación en Enseñanza de la Biología.

se puede identificar a un ser vivo, indicaron que se debe observar que sí cumpla con las funciones vitales y que esté formado por células. No obstante, se requiere profundizar en el significado de la función de relación y nutrición, especialmente en lo relacionado con las modificaciones del medio y al intercambio de energía (luz, calor, energía química).

\section{BIBLOGRAFÍA}

Gómez, A. (2014). Enseñanza de la biología basada en modelos y modelización: una introducción. Bio-grafía, Escritos sobre la Biología y su Enseñanza. 7 (13). 101107.

Gutiérrez, R. (2014). Lo que los profesores de ciencia conocen y necesitan conocer acerca de los modelos: aproximaciones y alternativas. Bio-grafía, Escritos sobre la Biología y su Enseñanza. 7(13), 37-67.

Jorba, J. y Sanmartí, N. (1996): Enseñar, aprender y evaluar: un proceso de regulación continua. Madrid: MEC.

Leach, J. \& Scott, P. (2002). Designing and evaluating science teaching sequences: An approach drawing upon the concept of learning demand and a social constructivist perspective on learning. Studies in Science Education. 38. 115-142.

Lopez, A. y Moreno, G. (2014). Sustentación teórica y descripción metodológica del proceso de obtención de criterios de diseño y validación para secuencias didácticas basadas en modelos: El Caso del fenómeno de la fermentación. Biografía, Escritos sobre la Biología y su Enseñanza, 7 (13). 108-126.

Pujol, R. (2003). Didáctica de las Ciencias en la Educación Primaria. Madrid, España: Síntesis educación. 